\title{
The chaotic solar cycle
}

\section{Analysis of cosmogenic ${ }^{14} \mathrm{C}$-data}

\author{
A. Hanslmeier ${ }^{1}$ and R. Brajša ${ }^{2}$ \\ 1 Inst. für Physik, Geophysik Astrophysik und Meteorologie, Univ.-Platz 5, 8010 Graz, Austria \\ e-mail: arnold.hanslmeier@uni-graz.at \\ 2 Hvar Observatory, Faculty of Geodesy, University of Zagreb, Kačićeva 26, 10000 Zagreb, Croatia \\ e-mail: romanb@geof.hr
}

Received 9 August 2009 / Accepted 18 September 2009

\section{ABSTRACT}

\begin{abstract}
Context. The study of solar activity over long time intervals using proxies.
Aims. The periodicity of the solar activity cycle is studied. The solar activity cycle is governed by a complex dynamo mechanism. Methods of nonlinear dynamics enable us to learn more about the regular and chaotic behavior of solar activity.

Methods. By applying methods of nonlinear dynamics, the solar activity cycle is studied by using solar activity proxies that have been reaching into the past for over 10000 years. The complexity of the system is expressed by several parameters of nonlinear dynamics, such as embedding dimension or false nearest neighbors, and the method of delay coordinates is applied to the time series.

Results. The solar activity cycle is found to be on the edge of chaotic behavior. This can explain the observed intermittent period of longer lasting solar activity minima. Filtering the data by eliminating variations below a certain period (the periods $380 \mathrm{yr}$ and $57 \mathrm{yr}$ were used) yields a far more regular behavior of solar activity.

Conclusions. The solar time series of solar activity proxies used here clearly shows that solar activity behaves differently from random data. The unfiltered data exhibit a complex dynamics requiring an embedding dimension $>15$. The variations become more regular when filtering the data. The results also indicate that solar activity proxies are influenced by other than solar variations and reflect solar activity only on longer time scales.
\end{abstract}

Key words. solar-terrestrial relations - Sun: activity - Sun: general

\section{Introduction}

The Sun is the only star where its activity cycle has been studied in detail and can be traced back in time over periods spanning several 10000 years by using proxies for solar activity. Such long time series are needed to answer the question whether the solar activity and the underlying dynamo mechanism is of periodic, chaotic or stochastic nature. In a chaotic system a strong sensitivity on initial conditions exists. For slightly different initial conditions (or perturbations of the system) the solutions diverge exponentially giving the impression of a stochastic behavior. A stochastic process is one whose behavior is nondeterministic in that a system's subsequent state is determined both by the process's predictable actions and by a random element. Up to now it has not been clear whether the solar dynamo behaves like a chaotic, or a stochastic system. Chaotic phenomena in astrophysics and cosmology, mainly for dynamics in the solar system and galactic dynamics, as well as applications to cosmology, such as properties of cosmic microwave background radiation, were reviewed by Gurzadyan (2002), while the phenomena showing evidence of the nonlinear dynamics in solar physics are summarized by Hanslmeier (1997).

Direct solar activity observations are only available for a few centuries and proxies such as observations of aurorae, cosmogenic isotopes, growth of certain plants like corals, etc. have to be used to obtain a longer time series of the solar activity. An overview of these proxies can be found, e.g., in Hanslmeier (2007).
Nonlinear effects in the solar activity have been studied in theoretical models and reviewed by several authors (Stix 1981; Ruzmaikin 1985; Hoyng 1990, 1992; Rosner \& Weiss 1992; Wilson 1994; Stix 2002; Ossendrijver 2003; Rüdiger \& Hollerbach 2004). In particular, the question of whether the solar cycle is an example of deterministic chaos has been examined in different papers based on theoretical considerations and data analysis (Schmalz \& Stix 1991; Jennings 1992; Ruzmaikin et al. 1992; Carbonell et al. 1994; Tobias 1996; Charbonneau 2005; Letellier et al. 2006; Volobuev 2006). Brandenburg \& Spiegel (2008) introduce on/off intermittency into a mean field dynamo model by imposing stochastic fluctuations in either the $\alpha$-effect (a measure for the effect of convection and coriolis forces) or through the inclusion of a fluctuating electromotive force. Smallscale fluctuations with time scales in the order of 0.3-3 years, which are sufficiently strong, can produce long-term variations in the system on time scales of hundreds of years. They find no suppression of magnetic activity in both solar hemispheres and point out that the relation between the level of solar magnetic activity and sunspot numbers may be never understood completely.

These theoretical models help to explain the observed periods of strongly reduced solar activity, as for the most often cited the Maunder minimum (1645-1715) which was, e.g., described by Eddy (1976). The solar activity was not zero during that period, and Ribes \& Nesme-Ribes (1993) presented results from observations of sunspots during that period made at the Paris observatory. 
Weiss (1988) studied secular solar and geomagnetic variations in the past 10000 years. Feynman \& Gabriel (1990) studied the 88 year cycle and the Maunder minimum and their conclusion was that there is evidence for a chaotically behaving Sun, because the solar dynamo is chaotic and is operating in a region close to the transition between period doubling and chaos. Since Maunder-type minima reoccur irregularly during the millennia, it appears that the Sun remains close to this transition to and from chaos. Tapping et al. (2007) developed a model indicating a lower mean irradiance during the Maunder minimum of $1 \pm 0.4 \mathrm{~W} \mathrm{~m}^{-2}$ lower than the mean irradiance over the last solar activity cycle.

The mean monthly sunspot index, for a period of 235 years was studied by Pavlos et al. (1992). The existence of a lowdimensional strange attractor was shown and a correlation dimension (about 4.5) was given. Spiegel (1993) and Spiegel \& Zahn (1996) propose that the solar butterfly diagram represents the motion of activity waves that are very stable nonlinear solitary waves. The waves are driven by an instability under the convection zone in a layer called the tachocline. Charbonneau (2001) studied one-dimensional maps to which dynamo equations can be reduced. A strong odd-even signal is found to be a good precursor to the transition from bursting to quiescent behavior.

Consolini et al. (2006) make a principal-components (PC) analysis of the solar cycle. Their main results can be summarized as: the main 11-year cycle can be described by only 2 modes, a full description of the chaotic nature of solar cycle, however, requires up to 28 modes. The Lyapunov exponents of the first 28 PCs are evaluated showing the existence of two different chaotic regimes.

Chaos and intermittency in the solar cycle were reviewed by Spiegel (2009). In this paper a description of the behavior of the solar cycle (both in time and spatial domain) by means of waves is suggested. If the solar activity cycle resembles a nonlinear dynamo, this can be approximated by a complex system of Lorenz equations (Jones et al. 1985). From a sixth order system of ordinary differential equations plane nonlinear dynamo waves can be obtained. These can model the activity of stars, and a sequence of bifurcations is observed that ends in chaos as the dynamo number $D$ is increased. Letellier et al. (2006) studied evidence of low-dimensional chaos in sunspot cycles.

Nonlinear dynamics methods have also been applied to predicting solar activity cycles (Sello 2001; Kitiashvili \& Kosovichev 2008) and distinguishing between a stochastic and a chaotic behavior is very important in this context (Duhau 2003; Yeates et al. 2008), especially for describing grand solar minima and maxima (Usoskin et al. 2007). Group sunspot number (GSN) values, averaged over decades, were reconstructed by Ogurtsov (2005) for a time interval 8505 BC-AD 1945 using data on the concentration of radiocarbon in tree rings in order to make prediction of an average level of solar activity. The result was that the average activity of the Sun during 2005-2045 would be lower than before.

It has to be stressed, however, that it is in principle still an open question whether the solar cycle is representing a chaotic or a stochastical system. Mininni et al. (2002) assume a nonchaotic solar cycle. Their analysis was based on temporal and latitudinal distribution of sunspots recorded since 1874, and they found evidence of the absence of low-dimensional chaos, that suggests that the spatiotemporal irregularities observed in the solar cycle come from the superposition of regular structures with a stochastic background. Charbonneau \& Dikpati (2000) studied fluctuations in a Babcock-Leighton model of the solar cycle and introduced large-amplitude stochastic fluctuations in either or both the meridional flow and poloidal source terms in the model. Solar cycle-like oscillatory behavior persists even for fluctuation amplitudes as high as $300 \%$.

In this paper we study a proxy for solar activity, the isotope ${ }^{14} \mathrm{C}$. This will enable us to use a long time series for the investigations. The abundance of this isotope depends on the cosmic ray flux, which is lower when the Sun is in its most active phase. There is an anticorrelation in the production of cosmogenic isotopes and solar activity. Geomagnetic field variations also cause irregularities in the ${ }^{14} \mathrm{C}$ data. To estimate possible short term geomagnetic influence, the data were convolved by random functions of different amplitudes.

\section{Data and data analysis}

\subsection{Data}

The sunspot number was reconstructed based on dendrochronologically dated ${ }^{14} \mathrm{C}$ radiocarbon data covering the past 11400 years (see Solanki et al. 2004). Our data consist of a series of reconstructed 10-year averaged sunspot numbers with their $68 \%$ uncertainty. Years are given BP (before present), i.e., the calendar AD year, Yad, is related to the BP year, Ybp, as $\mathrm{Yad}=1950$-Ybp. The data are available in tabular form. The tabulated years correspond to centers of the corresponding 10-year intervals. Negative values are artifacts and are consistent with zero within the error limits. These ${ }^{14} \mathrm{C}$ radiocarbon data are used for applying different methods of nonlinear dynamics.

As stated in the introduction, the variations seen in the data have not only a solar origin but also depend on geomagnetic field variations. The data were corrected for palaeogeomagnetic variations. However, to test the robustness of our results, field variations were simulated by adding random data of different amplitudes to the original values and then studying how the results changed.

\subsection{Methods of nonlinear dynamics}

\subsubsection{Time series analysis}

First we calculated the mutual information. To estimate the embedding dimension, $m$, the false nearest neighbors, FNN (see e.g. Rhodes \& Morari 1997) were calculated. From these results we could derive the appropriate embedding dimension $m$ that is needed to reconstruct the phase space and to plot delay coordinates. Our data set is given as a time series sampled at equal distant intervals. Such a system can be characterized by the embedding dimensions. The phase space of a system is made up of all coordinates and velocities (i.e. changes in the coordinates). The phase space of a given system describes its time evolution. The phase space of a time series can be reconstructed by the method of delays that is the most important phase space reconstruction technique.

Consider a series of measurements $X_{n}$. Such a series does not represent the multidimensional phase space of the dynamical system. One has to design some method to unfold the multidimensional structure of the data. This is done in the method of delays. From time-delayed values of the scalar measurements vectors in the new space, called the embedding space, we construct:

$X_{n}=\left(X_{n-(m-1) \tau}, X_{n-(m-2) \tau}, \ldots, X_{n}\right)$.

The number $m$ is called the embedding dimension, and $\tau$ is called the delay. The theorems of Takens (1980) and Sauer 

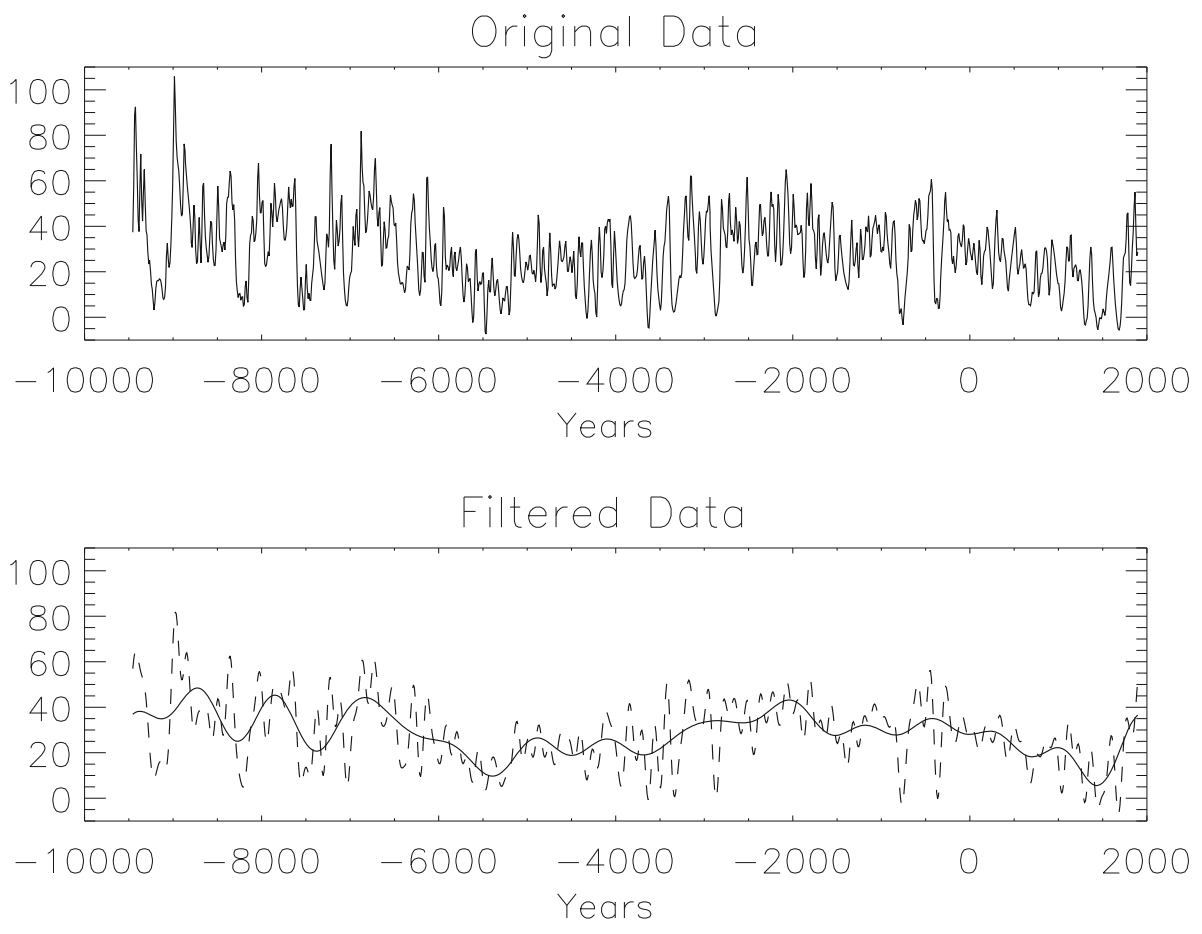

Fig. 1. The data series for solar activity proxies. On the upper plot the original data, on the lower plot, the Fourier-filtered data are shown. Fluctuations at periods for $t \leq 380 \mathrm{yr}$ (full line) and for $t \leq 57 \mathrm{yr}$ were suppressed by a Fourier filter.

(Sauer et al. 1991) state that, if the sequence $X_{n}$ consists of scalar measurements of the state of a dynamical system, then the time delay embedding provides a one-to-one image of the original set $X_{n}$ provided if $m$ is large enough. It has to be stressed, however, that these theorems technically deal with infinite, noise-free trajectories of a dynamical system and are not guaranteed to hold for finite series of measured data (Schreiber 1999). In our case we have to assume that our results are valid for the limited and not the noise-free time series.

Under these assumptions, if $N$ scalar measurements exist, then the number of embedding vectors is $N-(m-1) \tau$.

\subsubsection{False nearest neighbor estimation}

The first step in applying methods of nonlinear dynamics will be therefore to estimate $m$, the embedding dimension. One method that was developed is found in Kennel et al. (1992) and is called false nearest neighbors. The principle is simple. Suppose $m_{0}$ is the minimal embedding dimension for a time series $X_{i}$. In an $m_{0}$-dimensional delay space, the reconstructed attractor is a oneto-one image of the attractor in the original phase space. The neighbors of a given point are mapped onto neighbors in the delay space. The Lyapunov exponents determine the evolution of a dynamical system. Thus, the shape and neighborhood of points will be changed according to the Lyapunov exponents. However, neighborhoods will be mapped into neighborhoods again. The topological structure will be preserved. This is not the case, when $m<m_{0}$ is taken for the embedding. Here, the topological structure is no longer preserved, so points are projected into neighborhoods of other points to which they would not belong in higher dimensions. Such points are called false neighbors. A description of the false nearest neighbor method, FNN, is given by Rhodes \& Morari (1997).

Therefore, in the subsequent analysis we first calculate the embedding dimension by the method of false neighbors and then investigate the method of delays.

\subsubsection{Mutual information}

The mutual information (see, e.g., Kantz \& Schreiber 1997) is a generalization of the well known autocorrelation function. In information theory, mutual information is defined by

$I(X, Y)=\int_{X} \int_{Y} f_{X Y}(x, y) \log _{a} \frac{f_{X, Y}(x, y)}{f_{X}(x) f_{Y}(y)} \mathrm{d} x \mathrm{~d} y$,

where $X, Y$ denote two variables, $f_{X, Y}(x, y)$ is the joint probability density function of $X$ and $Y$, and $f_{X}(x), f_{Y}(y)$ are the probability density functions of $X$ and $Y$ respectively. The units of information $I(X, Y)$ depend on the base $a$ of the logarithm (usually $a=2)$. Assuming a partition of the domain of $X$ and $Y$ the double integral becomes a sum over the cells. For a time series $\left\{X_{t}\right\}_{t=1}^{n}$ sampled at fixed times $\tau_{\mathrm{s}}$, the mutual information is defined as a function of the delay $\tau$ assuming the two variables $X=X_{t}$ and $Y=X_{t-\tau}$, i.e., $I(\tau)=I\left(X_{t}, X_{t-\tau}\right)$.

An appropriate choice of time delay for the embedding is one such that the mutual information is at a minimum. More information can be found in the paper cited above.

\section{Results}

In the upper image of Fig. 1 the reconstructed sunspot data are shown and the original data are plotted and as mentioned the time interval between two data points is 10 years. To suppress noise, a Fourier filter was applied to eliminate the shorter time scale fluctuations seen in the data. In the lower image the filtered data are shown, and the Fourier filtering was adapted so as to filter out noise on two levels: a) for fluctuations shorter than 380 years (full line) b) for fluctuations shorter than 57 years (dashed line).

We simulated a possible variation in the geomagnetic field to demonstrate the robustness of the results. It should be stressed that the data were already corrected for known geomagnetic field variations. In Fig. 2 the results are shown for a simulation of random geomagnetic variations of a period twice the filtering period of 380 years. The amplitudes were chosen to simulate a worst 
Original Data + random

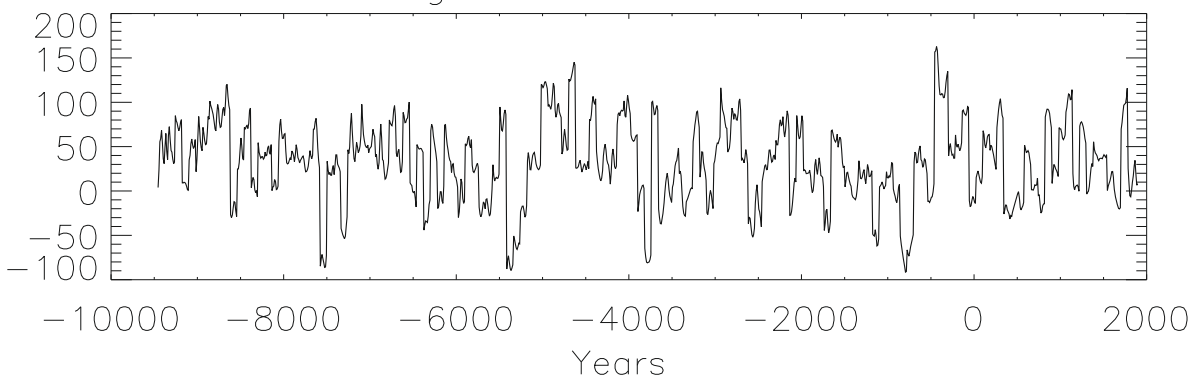

Filtered Data

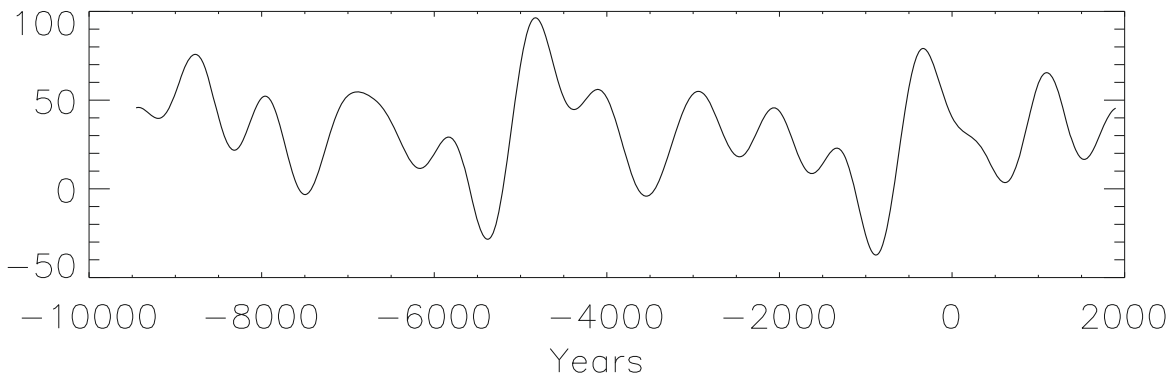

Fig. 2. The data series for solar activity proxies. To simulate geomagnetic field-strength variation, a random signal of similar amplitude to the original signal and a period of about 760 years were added. On the upper plot the original data, on the lower plot, the Fourierfiltered data are shown (in this case for filtering for $t \leq 380 \mathrm{yr}$ ).

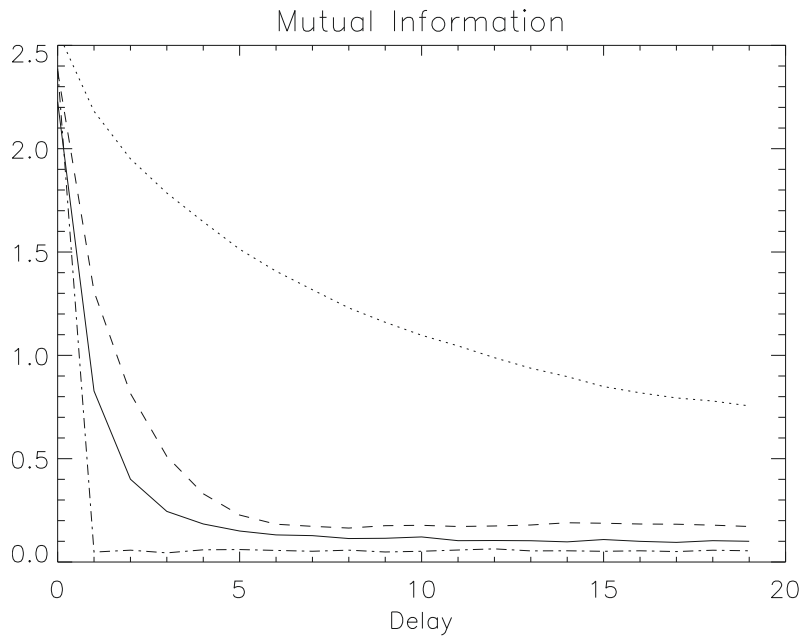

Fig. 3. Mutual information as a function of delay for original data (full line), for Fourier-filtered data $t \leq 380 \mathrm{yr}$ (dotted), $t \leq 57 \mathrm{yr}$ (dashed) and for random data (dashed dotted).

case which means they were of comparable amplitude than the original data. These assumptions correspond to the statements by Solanki et al. (2004, Fig. 3c), who reported a magnetic dipole variation of a factor of two at the maximum and of a period that is at least longer than the value used for our filtered data.

In Fig. 3 the results are shown for the mutual information. It is given as a function of the delay. The graphs are given for the original data, filtered data $(t<380 \mathrm{yr})$, filtered data $(t<57 \mathrm{yr})$, and random data. For the filtered data, the mutual information curve decreases less steeply than for the original data. For the random data the graph immediately declines to zero.

In Fig. 4 the results for the false nearest-neighbor calculations are shown. On the abscissa, the dimension is shown on the ordinate, the fraction of false nearest neighbors. For the original data the curve approaches zero at values of dimension $m>15$. For the filtered data $t<380 \mathrm{yr}$ the convergence to zero occurs at $m>14$, for the filtered data $t<57 \mathrm{yr}$ the convergence to zero

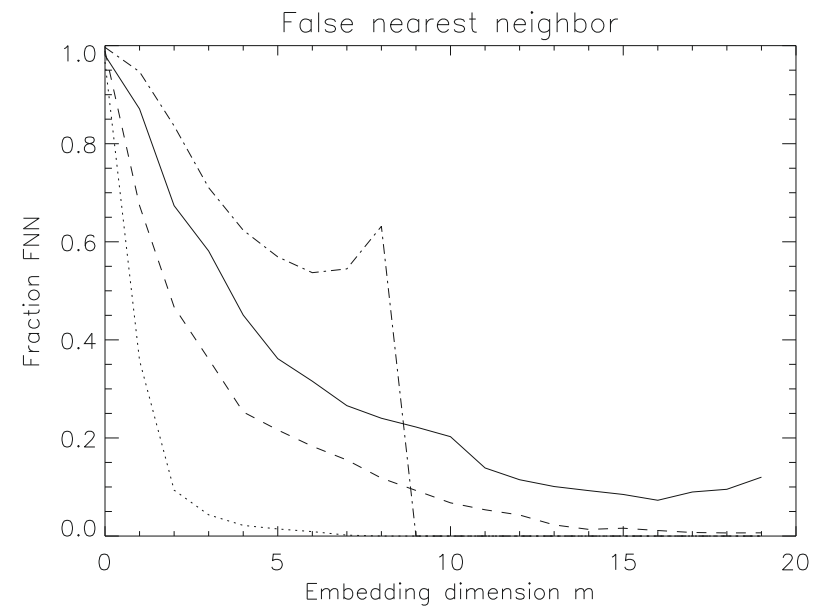

Fig. 4. False nearest neighbors for original data (full line), for Fourierfiltered data $t \leq 380 \mathrm{yr}$ (dotted), $t \leq 57 \mathrm{yr}$ (dashed), and for random data (dash dotted).

occurs at $m>15$. For comparison, the random data do not converge at all (the decline to zero at $m=9$ is an artefact of the finite series).

Next, the delay method was applied. This method is again based on the theorem of Takens (1981) and Sauer et al. (1991). The delays were selected for the following values $d=$ $1,5,10,15,20,25$. The results are given for the original data in Fig. 5, and in Fig. 6 the results for the filtered data $t \leq 380 \mathrm{yr}$ and in Fig. 7 for $t \leq 57 \mathrm{yr}$. Comparing these three figures, we see that the dynamics become more complex when going to smaller time-scale variations. In Fig. 8 the results are shown for random data.

The influence of geomagnetic disturbances is demonstrated in Fig. 9. As described above, the variation in the geomagnetic field is simulated. We only give here the results only for case of filtered data $(t<380 \mathrm{yr})$. The behavior of the attractor given by the delay coordinates does not show a significant difference with respect to the original filtered data. 
A. Hanslmeier and R. Brajša: The chaotic solar cycle. I.
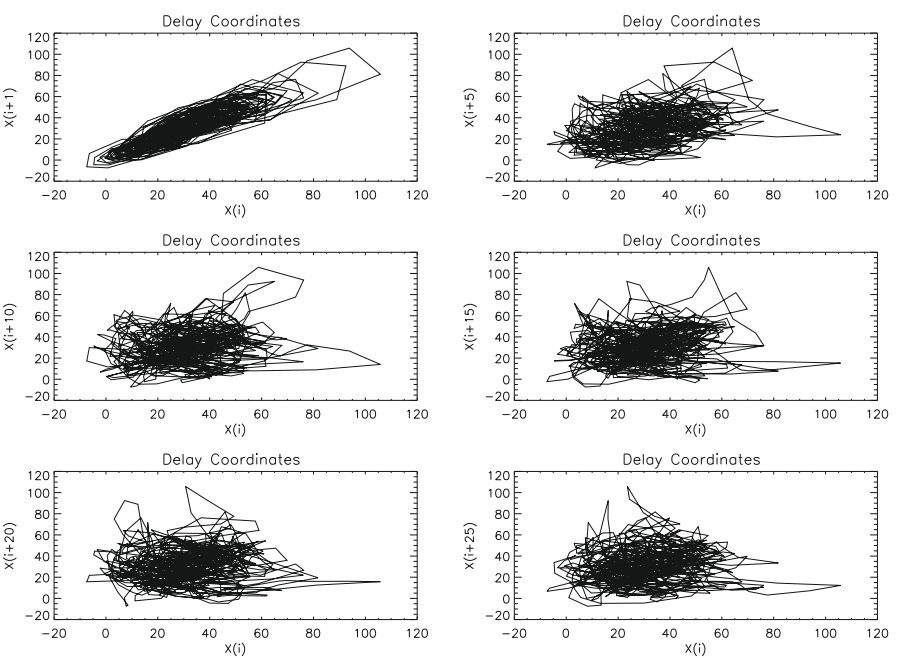

Fig. 5. Method of delays applied to original data.
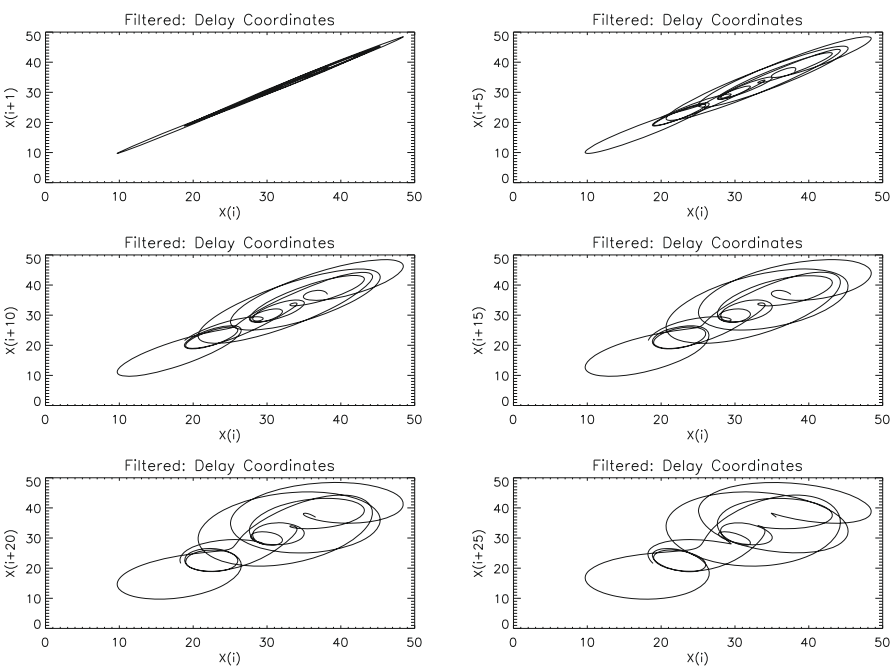

Fig. 6. Method of delays applied to filtered data $(t \leq 380 \mathrm{yr})$.

\section{Discussion and conclusions}

We applied standard methods of nonlinear dynamics to study the behavior of solar activity proxies. It has to be stressed that the data consists of averaged values where the averaging was done over a 10 year interval. Therefore, the results are only indicative of longer term variations and do not include and represent the 11 year solar activity cycle because of the sampling rate of 10 years. Therefore, the conclusions to be taken are valid for longer time-scale modulations of the 11 year cycle. To make a further distinction between shorter time scale and longer timescale variations a Fourier filtering was applied. By such a filter, variations below a time scale of 57 years were eliminated.

The mutual information and false nearest neighbor methods applied give insight into the complexity of the underlying system. From this information we estimated the embedding dimension $m$. In the case of the unfiltered data, we obtained the value $m=15$. At this value the number of false nearest neighbors becomes very small (Fig. 4). In the case of filtered data this value is lower, for the elimination of fluctuations with $t \leq 380 \mathrm{yr}$ the value is about 5 , for the elimination of fluctuations with $t \leq 57 \mathrm{yr}$ the value is around 10 . The complexity of the system therefore strongly increases on shorter timescales. Generally, the time series of proxies exhibits a complex structure and a good estimate
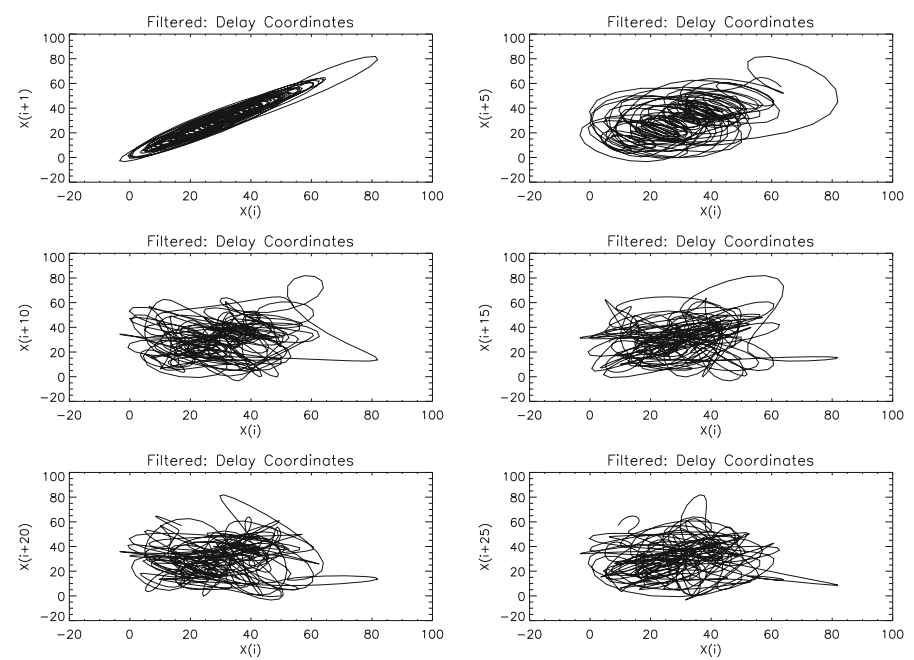

Fig. 7. Method of delays applied to filtered data ( $t \leq 57 \mathrm{yr})$.
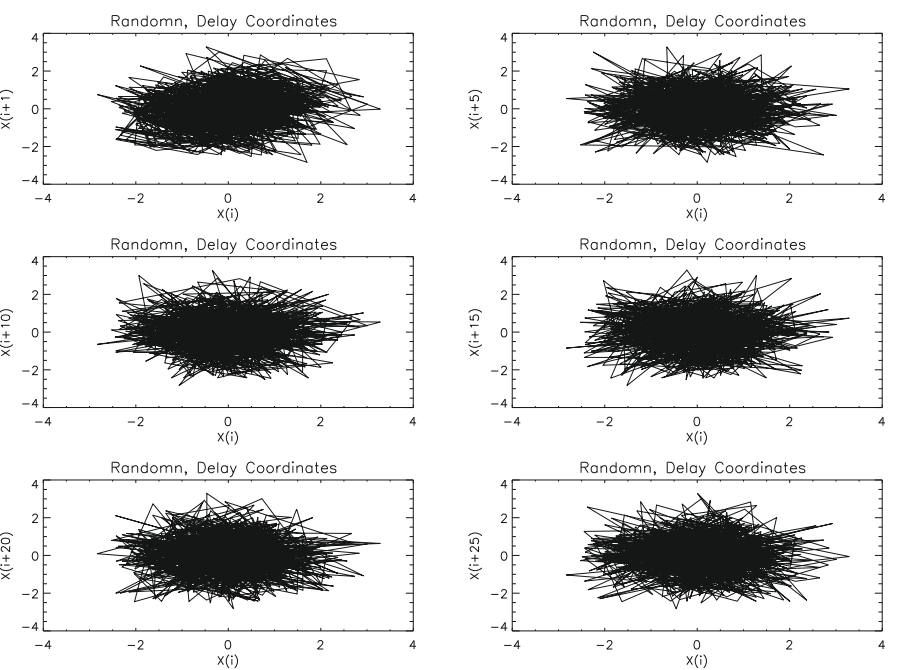

Fig. 8. Method of delays applied to random data.

for $m$ is required to reveal the topologically correct behavior by applying the method of delay coordinates.

In Figs. 5-7 the results for the delay coordinates are given for different delay values. It is seen that the topological structure is quite complex when considering the unfiltered original data (Fig. 5). The structure, however, becomes quite simple when considering the filtered data, eliminating $t \leq 380 \mathrm{yr}$ fluctuations (Fig. 6). The case where fluctuations on time-scales $t \leq 57 \mathrm{yr}$ have been eliminated is between the two cases mentioned before. This can be interpreted in the sense that solar activity proxies seem to exhibit a more regular and predictable behavior when the variations at larger time scales are considered. These larger time scales are well above 100 years.

Comparing our results for the sunspot index proxies with the artificially generated random time series (dashed dotted line in Figs. 3 and 4) we clearly can state the following.

- Some structure appears even in the unfiltered data; the system behaves like a system of high complexity with an embedding dimension over 15 .

- Considering the two cases of filtered data, we clearly see that the appearance of the delay plots become less complex. This is also reflected by the fact that the mutual information 

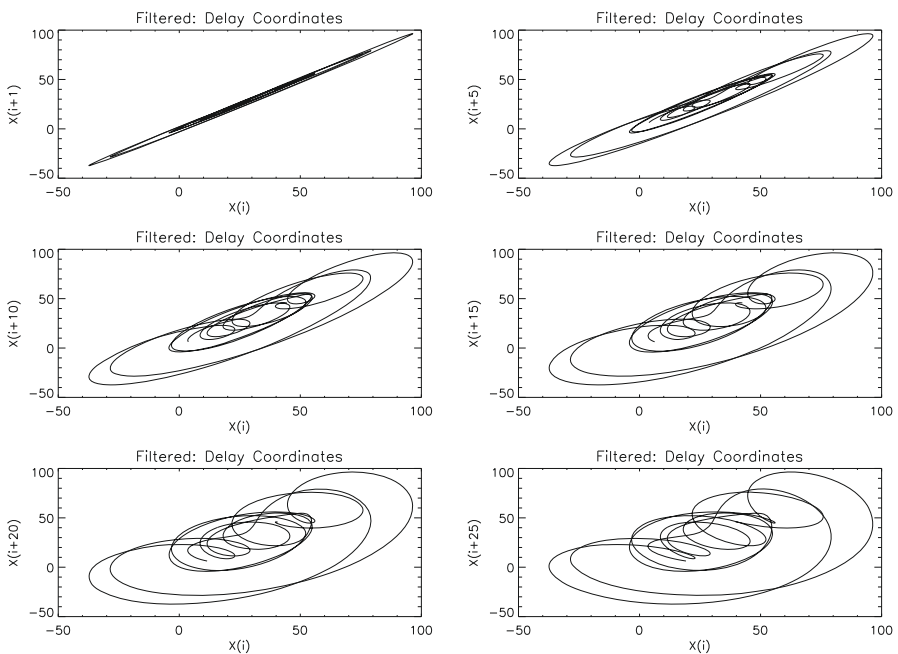

Fig. 9. Method of delays applied to filtered data $(t<380 \mathrm{yr})$ and added a noise component to simulate possible variations of the cosmogenic flux due to geomagnetic variations.

becomes greater than 10 when eliminating fluctuations below 57 year periods.

- The solar activity proxies clearly behave differently from random data.

- The analysis suggests a better understanding of the behavior on larger time-scales, but this may also be because we only used solar activity proxies and not a solar activity parameter itself.

Of course we have to take into account that the cosmic ray flux that generates the ${ }^{14} \mathrm{C}$ isotope is modulated by two factors: (i) the solar magnetic field, and therefore the solar activity in general, and (ii) the variations of the Earth's magnetic field. The time interval from the present to 10-12 ka ago of these variations was studied in the paper of Dergachev et al. (2006). The Earth's magnetic field can be approximated by two components: an axial and a geocentric dipole. The remaining non-dipole field is much smaller is and a regional feature. Large changes within a few ka can occur on these scales. Variations in the intensity of the Earth's magnetic field were studied by Jacobs (1998).

The influence of the noise due to geomagnetic variations on our data may also explain why the embedding dimension found here seems to be much higher than the value that was given, e.g., by Spiegel (1993). Such a low value can only be reached when a filtering is applied (eliminating fluctuations $t \leq 380 \mathrm{yr}$ ). This result clearly demonstrates that solar activity proxies, while correlated with solar activity parameters, have to be used with caution and may only reflect variations in solar activity over several activity cycles.

The influence of geomagnetic field variations was simulated by overlaying random functions of different amplitudes on the original data, and our results seem to be quite robust. The geomagnetic poloidal field intensity depends on geodynamic processes extending (plate reorganizations at the surface, increases in the vigor of outer core convection). Because of such processes, supercontinents have appeared throughout the Earth's history. Several major long-term changes seem to exist s due to these processes: 400-350, 350-250, 250-175, and 175-10 Ma (Biggin \& Thomas 2003), but all these are beyond the scope of our data sets, of course. Power spectra of both paleointensity and inclination variations have peaks at about $100 \mathrm{kyr}$ periods, which is still far above the length of the time series considered
(Yamazaki et al. 2008). The data used here have been corrected for palaeomagnetic variations (Solanki et al. 2004). Based on the values given in that work, the influence of geomagnetic variations can be estimated by convolving the data with random numbers as described above.

We will extend these investigations to other proxies of solar activity.

Acknowledgements. We thank Dr. I. Usoskin for giving us the ${ }^{14} \mathrm{C}$ radiocarbon data. This work is sponsored by the Air Force Office of Scientific Research, Air Force Material Command, USAF, under grant number FA8655-07-1-3093. Also, the research leading to the results presented in this paper has receives partial funding from European Community's Seventh Framework Programme (FP7/2007-2013) under grant agreement No. 218816. Finally, the authors acknowledge the support from the Austrian-Croatian Bilateral Scientific Project (2008/08) for financing the exchange of scientists. We also want to thank an unknown referee for helpful comments and suggestions.

\section{References}

Brandenburg, A., \& Spiegel, E. A. 2008, Astron. Nachr., 329, 351

Biggin, A. J., \& Thomas, D. N. 2003, Geophys. J., 152, 392

Carbonell, M., Oliver, R., \& Ballester, J. L. 1994, A\&A, 290, 983

Charbonneau, P. 2001, Sol. Phys., 199, 385

Charbonneau, P. 2005, Sol. Phys., 229, 345

Charbonneau, P., \& Dikpati, M. 2000, AJ., 543, 2, 1027

Consolini, G., Tozzi, R., \& de Michelis, P. 2006, AGU Fall Meeting Abstracts, A327

Dergachev, V. A., Dmitriev, P. B., Raspopov, O. M., \& Jungner, H. 2006, Geomagnetism and Aeronomy, 46, 118

Duhau, S. 2003, Sol. Phys., 213, 203

Eddy, J. A. 1976, Science, 192, 1189

Feynman, J., \& Gabriel, S. B. 1990, Sol. Phys., 127, 393

Gurzadyan, V. G. 2002, in The Ninth Marcel Grossmann Meeting, ed. V. G. Gurzadyan, R. T. Jantzen, \& R. Ruffini, 182

Hanslmeier, A. 1997, Hvar Obs. Bull., 21, 77

Hanslmeier, A. 2007, The Sun and Space Weather, 2nd edn. (Dordrecht: Springer-Verlag)

Hoyng, P. 1990, in Solar Photosphere: Structure, Convection, and Magnetic Fields, ed. J. O. Stenflo, 359

Hoyng, P. 1992, in The Sun: A Laboratory for Astrophysics, ed. J. T. Schmelz, \& J. C. Brown (Dordrecht: Kluwer Academic Publishers), NATO ASI Ser., 373,99

Jacobs, J. A. 1998, Surv. Geophys., 19, 139

Jennings, R. L. 1992, in The Solar Cycle, ed. K. L. Harvey, ASP Conf. Ser., 27, 543

Jones, C. A., Weiss, N. O., \& Cattaneo, F. 1985, Physica D, 14D, 161

Kantz, H., \& Schreiber, T. 1997, Nonlinear Time Series Analysis (Reading, Massachusetts: Cambridge University Press)

Kennel, M. B., Brown, R., \& Abarbanel, H. D. I. 1992, Phys. Rev. A, 45, 3403

Kitiashvili, I., \& Kosovichev, A. G. 2008, ApJ, 688, L49

Letellier, C., Aguirre, L. A., Maquet, J., \& Gilmore, R. 2006, A\&A, 449, 379

Mininni, P. D., Gómez, D. O., \& Mindlin, G. B. 2002, Phys. Rev. Lett., 89, 061101

Ogurtsov, M. G. 2005, Sol. Phys., 231, 167

Ossendrijver, M. 2003, A\&AR, 11, 287

Pavlos, G. P., Dialetis, D., Kyriakou, G. A., \& Sarris, E. T. 1992, Ann. Geophys., 10,759

Rhodes, C., \& Morari, M. 1997, Phys. Rev. E., 55, 6162

Ribes, J. C., \& Nesme-Ribes, E. 1993, A\&A, 276, 549

Rosner, R., \& Weiss, N. O. 1992, in The Solar Cycle, ed. K. L. Harvey, ASP Conf. Ser., 27, 511

Rüdiger, G., \& Hollerbach, R. 2004, The Magnetic Universe (Weinheim: Wiley$\mathrm{VCH}), 95$

Ruzmaikin, A. A. 1985, Sol. Phys., 100, 125

Ruzmaikin, A., Feynman, J., \& Kosacheva, V. 1992, in The Solar Cycle, ed. K. L. Harvey, ASP Conf. Ser., 27, 547

Sauer, T., Yorke, J. A., \& Casdagli, M. 1991, J. Stat. Phys., 65, 579

Schmalz, S., \& Stix, M. 1991, A\&A, 245, 654

Schreiber, Th. 1999, Phys. Rep., 308, 1

Sello, S. 2001, A\&A, 377, 312

Solanki, S. K., Usoskin, I. G., Kromer, B., Schüssler, M., \& Beer, J. 2004, Nature, 431, 1084 
A. Hanslmeier and R. Brajša: The chaotic solar cycle. I.

Spiegel, E. A. 1993, in Columbia Univ. Annual Report, ed. C. Martin Spiegel, E. A. 2009, SSRV, 144, 25

Spiegel, E. A., \& Zahn, J.-P. 1996, in Columbia Univ. Annual Report, ed. M. Ruderman

Stix, M. 1981, Sol. Phys., 74, 79

Stix, M. 2002, The Sun, 2nd edn. (Berlin: Springer-Verlag), 364

Takens, F. 1980, Detecting Strange Attractors in Turbulence, in Dynamical Systems and Turbulence, Warwick, ed. D.A. Rand, \& L.-S. Young, LNM898 (Springer)

Tapping, K. F., Boteler, D., Charbonneau, P., et al. 2007, Sol. Phys., 246, 309
Tobias, S. M. 1996, A\&A, 307, L21

Usoskin, I. G., Solanki, S. K., \& Kovaltsov, G. A. 2007, A\&A, 471, 301

Volobuev, D. 2006, Sol. Phys., 238, 421

Weiss, N. O. 1988, in Secular Solar and Geomagnetic Variations in the Last 10000 Years, 69

Wilson, P. R. 1994, Solar and Stellar Activity Cycles (Cambridge: Cambridge University Press, 228)

Yamazaki, T., Kanamatsu, T., Mizuno, S., Hokanishi, N., \& Gaffar, E. Z. 2008, Geophys. Res. Lett., 35, 20, L20307

Yeates, A. R., Nandy, D., \& Mackay, D. H. 2008, ApJ, 673, 544 